\title{
Assessing the Relationship between ITIL Implementation Progress and Firm Size: Evidence from Malaysia
}

\author{
Kanagi Kanapathy (Corresponding author) \\ Faculty of Business and Accountancy, University of Malaya \\ Kuala Lumpur 50603, Malaysia \\ E-mail: kanagik@um.edu.my \\ Kashif Ibadullah Khan \\ Scope International Sdn. Bhd \\ Technology Park Malaysia, Bukit Jalil \\ Kuala Lumpur 57000, Malaysia \\ E-mail: kashif.um@gmail.com
}

Received: July 28, 2011

Accepted: September 26, $2011 \quad$ Published: January 16, 2012

doi:10.5539/ijbm.v7n2p194

URL: http://dx.doi.org/10.5539/ijbm.v7n2p194

\begin{abstract}
ITIL (Information Technology Infrastructure Library) is a service management framework that is considered as a set of best practices that assist organizations to increase IT service management effectiveness, service delivery and IT governance. ITIL enables technology departments to demonstrate strong IT service management via systematic execution of operations with stringent controls. A questionnaire survey method was utilized to explore the relationship between ITIL implementation progress and firm size, amongst 84 Multimedia Super Corridor (MSC) status firms in Malaysia. The study revealed that ITIL is widely adopted in MSC status firms. ITIL implementation progress was found to be positively associated with firm size, in terms of turnover, total number of employees and total number of IT staff.
\end{abstract}

Keywords: ITIL, IT service management, ITIL implementation, IT governance, Firm size

\section{Introduction}

Information technology (IT) has progressed from a conventional role to a business enabler within an organization. Information technology has now transformed into a most important organizational resource that any organization needs to manage (Henderson and Venkatraman, 1993). Information technology services are now a strategic asset and organizations are driven to invest appropriate resources into development, support and management of IT services. Organizations with wider level of IT investment noticed escalating performances, gauged by return on investment, sales per employee, return on sales and sales by total assets (Mahmood and Mann, 1993).

Businesses expect IT departments to act as a key enabler of more disciplined and systematic provisioning of IT services (Johnson and Hately, 2007). Information technology departments are expected to respond immediately to accommodate changing business landscape, capture new business opportunities and demonstrate strong and responsible financial management.

The growing challenge for IT professionals is to effectively coordinate and build strategic partnership with business, to deliver first-rate IT services. This level of commitment can only be achieved via systematic execution of operations with stringent controls of IT management. The swift movement towards service oriented organization model offers unparalleled prospects to business. This is where IT Infrastructure Library (ITIL) plays a vital role - to align business and technology service objectives.

Despite the growing importance and acceptance of IT Service Management (ITSM) and ITIL, there is a lack of literature on the implementation of ITSM frameworks (Hochstein, Zarnekow and Brenner, 2005). Not all organizations implementing ITIL fully benefited from ITIL implementation (Cater and Tan, 2005) as ITIL framework does not offer straightforward implementation approaches. The implementation mechanism is left to 
the implementer to decide. This research explores ITIL implementation in Malaysia and the relationship between different organizational factors and ITIL implementation progress.

This exploratory research was driven by the following research questions:-

Is ITIL implementation progress associated with firm size, in terms of annual turnover?

Is ITIL implementation progress associated with firm size, in terms of total number of employees?

Is ITIL implementation progress associated with firm size, in terms total number of IT staff in firm?

\section{ITIL and Organizational Factors}

As the role of services continue to increase in the global economy, effective IT management transformed into a competitive advantage for service providers. Different standards with structured approaches for delivering value and managing risk with governance functions have been developed. From technology operations to corporate strategies, IT standards are vital in organizations and IT is no longer recognized as a collection of hardware and software but is positioned as a service. Technology functions are modeled and managed as services via management frameworks. IT service management models enable organizations to reduce cost and governance risks and facilitate smooth change management.

ITIL is a standard framework for IT Service Management (ITSM). It is a set of best practices for managing and delivering ITSM. The framework describes the goals and activities of different processes that can be incorporated within IT organization (The Office of Government Commerce, 2004). IT organizations are now recognizing that standards improve productivity and business/IT synergies. These improvements lead to cost savings. Standards provide the ability for firms to rapidly incorporate new hardware/software into existing infrastructure.

IT service delivery accounted for approximately 70 to 90 percent of total cost of IT ownership (Fleming, 2005). IT service providers cope with a challenge to enhance the quality of IT services in a highly complex IT environment. This pressure demands strong collaboration from both external and internal service providers (Yan, Shen and Chen, 2010). Solution based on ITIL service management framework enable organizations to transform IT service management into a vital strategic asset. Efficient management of IT services has been positioned as a critical factor to the success of enterprise strategy.

ITIL is a set of best practices. However, the framework recommends no standards for the implementation sequence. By inducing such elasticity, ITIL implementation is transformed to a strategic management domain. Factors such as organization size, IT department size and other organizational factors have a propensity to play an important role in ITIL implementation. ITIL exemplify best practices, but it does not articulate solutions. ITIL does not stipulate a 'one size fits all' solution to the design and implementation of ITSM processes. ITIL framework outlines a structured approach for IT service management and service delivery processes. It does not matter if it is an internal IT organization or external service provider, each organization should adopt the guidelines of ITIL according to their organization context (IT Service Management Forum, 2004). It has been reported that the major benefit from ITIL implementation is improved quality of IT services, which is only made possible by elevated efficiency, standardization, process optimization and transparency via continuous process monitoring (Hochstein, Zarnekow and Brenner, 2005).

A study by Davis, Gillies, Smith and Thompson (1993) indicate that the size of the IT team or software development team is associated with software process improvement. A survey by Nerney (2003) revealed that a higher percentage of IT managers of large firms $(24 \%)$ were familiar with ITIL/ITSM compared to the IT managers in small and medium-sized firms (17\%). Previous research findings suggested that size of the IT team plays a role in process improvement [Davis, Gillies, Smith and Thompson (1993), Nerney (2003)]. This research attempted to examine the relationship between ITIL implementation progress and firm size in terms of turnover, number of staff and number of IT staff.

MSC (Multimedia Super Corridor) status is the recognition by the Malaysian Government through Multimedia Development Corporation for companies that actively participate and undertake ICT activities in MSC Malaysia. MSC status is awarded to both local and foreign companies that develop or use multimedia, IT and other high tech technologies to generate or enhance products and services. Majority of the MSC status organizations in Malaysia are large organizations and approximately $8 \%$ of these organizations are classified by MDC (Multimedia Development Corporation) as 'world class' organizations and world class incubators. Due to the size and complexity, these organizations require more discipline, governance and controls to manage the delivery of mission critical IT services.

This research investigates the following hypothesis:-

$\mathrm{H}_{1}$ : ITIL implementation progress is positively associated with firm size in terms of annual turnover 
$\mathrm{H}_{2}$ : ITIL implementation progress is positively associated with firm size in terms of total number of employees

$\mathrm{H}_{3}$ : ITIL Implementation progress is positively associated with firm size in terms of total number of IT staff

\section{Research Methodology}

As there is a lack of literature on ITIL implementation progress and success factors in the ITIL implementation domain [Hochstein, Zarnekow and Brenner (2005) and Potgieter, Botha, and Lew (2005)], an exploratory research method was selected. Exploratory research is performed when few or no earlier studies are available. There are no academic studies or literature available for ITSM/ITIL implementation progress in MSC status organizations in Malaysia. The focal point is to get insights and familiarity via exploratory research methods.

A questionnaire survey method was employed to gather data. The questionnaire was adopted from an Australian ITIL implementation research conducted by Cater and Tan (2005a, 2005b). Their research instrument had already been validated for reliability as well as content and construct validity. The same instrument was used by IT Service Management Forum (itSMF) Australia and Cater, Toleman and Tan (2006). Cater and Tan (2005a, 2005b) endorsed the use of their questionnaire in this study. A pilot study involving ten IT and technology managers was conducted and there were no significant changes made to the survey instrument.

The actual survey was conducted using online survey and email. The survey form was distributed to MSC status companies, targeting one respondent per company. The targeted respondents were limited to Chief Information Officers (CIOs), Chief Operations Officers (COOs), technical managers, business service managers, Process managers, IT capacity managers, Availability managers, IT service level managers, IT support managers, Release managers, Application/account managers, project managers, Information security managers, Project managers, ITIL/ITSM consultants and other streams of IT managers.

The sample size of this research was 260 MSC organizations, with an expected response rate of $25 \%$ to $30 \%$. The selection of respondent organizations was done using stratified sampling method as it provides greater precision than a simple random sample of the same size. The approach for sample selection was proportionate stratification. In proportionate stratification, the sample size of each stratum is proportionate to the population size of the stratum. The potential respondent organizations were selected by using the Multimedia Development Corporation's (MDC) classification of organizations. The MDC classified organizations into software development, hardware design, internet based business services, creative multimedia and design, IT support services and shared service categories.

The final response rate of this survey was 33 percent i.e. 84 respondent firms. This response rate is higher than the response rate of $15 \%$ to $20 \%$ reported in Malaysian based surveys (Kanapathy and Jabnoun, 1998, Sohail and Teo 2003). The 84 respondents included Chief information officers (CIOs), Head of IT Departments, IT Managers, technology service managers, technology service delivery managers and senior IT operation managers. Descriptive statistics were used to transform data into meaningful summaries to understand, interpret and provide descriptive information. Normality test indicated that the data is not normally distributed. Hence, Spearman's rank correlation coefficient was employed to measure statistical dependence between two variables and to assess how well and in depth the relationship between two variables can be described. The Spearman's Rho correlation illustrates the magnitude and direction of the association between two variables.

\section{Findings}

The respondent companies were from various sectors; shared services and outsourcing (26.53\%), software development (23.48\%), IT support services (17.35\%), computer and electronic manufacturing (11.22\%), internet based business services $(9.18 \%)$ creative multimedia and design $(5.10 \%)$ and others $(7.14 \%)$. Among these 84 respondent organizations, $54.02 \%$ are local owned private limited companies, $21.84 \%$ are foreign owned and $24.14 \%$ are local-foreign joint ventures.

The results revealed that ITIL implementation is progressing in MSC status companies - $41 \%$ of respondent firms have largely or fully implemented ITIL framework. Only $16 \%$ of the respondent firms have no plan to implement ITIL. This is in accordance with the previous research findings that more and more organizations are now implementing ITIL as the de-fecto standard of IT service management (Cater, Toleman and Tan, 2006).

\subsection{ITIL Implementation Progress and firm size in terms of annual turnover}

Based on the Spearman's correlation test, a week positive relationship between firms' annual turnover and ITIL implementation progress was identified. The Spearman's correlation coefficient of 0.288 , as shown in Table 1 indicates a weak positive relationship. Hence, the result supports the hypothesis that for MSC status firms, ITIL implementation progress is positively associated with firms' annual turnover 


\subsection{ITIL Implementation Progress and firm size in terms of total number of employees}

As shown in Table 2, the Spearman's correlation coefficient of 0.523 clearly indicates a strong positive relationship between ITIL implementation progress and firm size in terms of total number of employees.

This positive relationship supports earlier research findings that there is a significant positive relationship between ITIL implementation progress and firm size in terms of total number of staff (Cater and Tan, 2005a). Another study by Ghobadian and Gallear (1997) reported that compared with the large organizations, SMEs are slower in implementing frameworks like Total Quality Management (TQM).

The results suggest that large MSC status firms are making more progress in ITIL implementation than small and medium-sized MSC status firms.

\subsection{ITIL Implementation Progress and firm size in terms of total number of IT staff in firm}

Spearman's correlation coefficient of 0.555 in Table 4.6 indicates a significant and strong positive relationship between number of IT staff in firm and implementation progress of ITIL. The results of this study further substantiate the findings reported in earlier research (Cater and Tan, 2005a) that organizations with a higher number of IT staff have made more progress toward ITIL implementation as compared to the organizations with lesser number of IT staff. This is also in line with Narney's (2003) finding, that a higher proportion of IT managers of large organizations $(24 \%)$ were familiar with ITIL compared to IT managers at small and medium-sized organizations.

\section{Conclusion and Recommendations}

ITIL service management framework stresses on IT processes to improve productivity, governance and agility to respond to changing market and business needs. Firms around the world started to adopt ITIL to accomplish business aspirations and goals. ITIL is a set of best practices and organizations need to consider the organizational factors that will be able to shape a winning ITIL implementation.

This research established that in MSC status firms:

ITIL implementation progress is positively associated with firm size, in terms of annual turnover.

ITIL implementation progress is positively associated with firm size, in terms of total number of employees.

ITIL implementation progress is positively associated with firm size, in terms of total number of IT staff.

In summary, this research establishes a reference point to gauge the level of penetration and ITIL implementation progress in MSC status firms. This study highlighted that firm size in terms of annual turnover, total number of employees and total number of IT staff, are important factors for ITIL implementation progress. Large MSC status firms are making better progress in implementing ITIL framework, compared to small and medium-sized firms.

\section{Implications}

This study makes significant contribution as this is the first study in the Malaysian context to gauge ITIL implementation progress. This will be a reference for future studies. This research provides evidence of ITIL implementation progress in MSC status firms and this will add to the body of knowledge on ITIL implementation. The results of this study will enable ITIL practitioners, IT Managers, CIOs, different streams of IT Managers and ITIL consultants to understand issues related to ITIL implementation, in terms of firm size.

The level of awareness need to be escalated among the CIOs and IT managers, as ITIL enable IT firms to deliver IT services with agility and quality while keeping the cost and risk at lower level. Adequate implementation of ITIL or ITSM framework can provide competitive advantages to organizations. ITIL is a set of best practices, but this does not mean that best practices lead to best implementation practices. Managers need to holistically understand the ITIL framework, its implementation success factors and align ITIL with the organizations' technology and service objectives. ITIL need to be embedded into technology, operations, process and procedures of organizations. There is a need for practitioners to study how ITIL can be implemented better in small and medium-sized firms.

\section{Limitations and Future Research}

There are limitations to this study. The data collected was only from MSC status firms (both local and foreign owned) and these findings may not be generalized to all Malaysian organizations. This research employed statistical measures to test hypothesis. Further qualitative research and case studies can be conducted to gauge best practices in ITIL implementation.

\section{References}

Cater-Steel, A., \& Tan, Wui Gee. (2005a). Implementation of IT Infrastructure Library (ITTL) in Australia: 
Progress and Success Factors. Proceedings of the IT Governance International Conference. Auckland.

Cater-Steel, A., \& Tan, Wui-Gee. (2005b). Summary of ITIL Adoption Survey Responses. Technical Report. itSMF Australia 2005 Conference: Toowoomba, Australia.

Cater-Steel, A., Toleman, M., \& Wui Gee Tan. (2006). Transforming IT service management - the ITTL impact. Proceedings of the 17th Australasian Conference on Information Systems. Adelaide.

Davis, C. J., Gillies, A. C., Smith, P., \& Thompson, J. B. (1993). Current practice in software quality and the impact of certification schemes. Software Quality Journal, pp.145-161. http://dx.doi.org/10.1007/BF00402266

Fleming, W. (2005). Using Cost of Service to Align IT. Presentation at itSMF .Chicago.

Ghobadian, A., \& Gallear, D. (1997). TQM and organization size. International Journal of Operations \& Production Management, 17 (2). pp.121 - 163.

Hochstein A., R. Zarnekow \& W. Brenner. (2005). Evaluation of service-oriented IT management in practice. Proceedings of International Conference on Services Systems and Services Management, vol.1, pp.80-84 .

IT Service Management Forum. (2004). Management forum: an introductory overview of ITIL. [Online] Available: http://ww.itsmfusa.org (December 17, 2010)

J.C. Henderson \& N. Venkatraman. (1993). Strategic alignment: Leveraging information technology for transforming organizations. IBM Systems Journal Archive, Volume: 32, Issue: 1, pp. 472-484. http://dx.doi.org/10.1147/sj.382.0472

Kanapathy K., \& Jabnoun, N. (1998). Are ISO 9000 and TQM Programs Paying Off for Malaysian Manufacturing Companies? Malaysian Management Review, 33(2). pp.40-46.

M.A. Mahmood \& G. J. Mann. (1993). Impact of information technology investment: An empirical assessment. Accounting, Management and Information Technologies, 3(1). January-March. pp.23-32. http://dx.doi.org/10.1016/0959-8022(93)90007-S

Mark W. Johnson, Andrew Hately \& Brent A. Miller Robert Orr. (2007). Evolving standards for IT service management. IBM Systems Journal, 46(3): 583-598. http://dx.doi.org/10.1147/sj.463.0583

Nerney,C. (2003). Survey: ITSM Needs to Pick Up Mindshare. [Online] Available: http://www.itmanagement.earthweb.com/service (December 15, 2010)

Potgieter, B. C., Botha, J. H., and Lew, C. (2005). Evidence That Use of the ITIL Framework is Effective. Tauranga, New Zealand: Proceeding of the 18th Annual Conference of the National Advisory Committee on Computing Qualifications.

Sohail, M.S., \& Teo Boon Hoong. (2003). TQM practices and organizational performances of SMEs in Malaysia: Some empirical observations. Benchmarking: An International Journal, 10 (1). pp.37 - 53

The Office of Government Commerce. (2004). An Introduction to ITIL. The Stationery Office. ISBN0113310617

Wei Yan, Beijun Shen \& Delai Chen. (2010). Supporting Distributed Collaboration in IT Service Management. Second International Workshop on Education Technology and Computer Science, vol. 2, pp.243-246.

Table 1. Correlation: ITIL implementation progress and firms' annual turnover

\begin{tabular}{|l|l|l|c|c|}
\hline \multicolumn{2}{|l|}{ Correlation } & & \multicolumn{1}{|c|}{$\begin{array}{c}\text { Annual } \\
\text { Turnover }\end{array}$} & $\begin{array}{c}\text { ITIL } \\
\text { Implementation } \\
\text { Progress }\end{array}$ \\
\hline $\begin{array}{l}\text { Spearman's } \\
\text { rho }\end{array}$ & $\begin{array}{l}\text { Annual } \\
\text { Turnover }\end{array}$ & $\begin{array}{l}\text { Correlation } \\
\text { Coefficient }\end{array}$ & 1.000 & $.288^{* *}$ \\
\cline { 3 - 5 } & Sig. (1-tailed) & & .004 \\
\hline **. Correlation is significant at the 0.01 level (1-tailed). \\
Spearman's rho $=0.288, \mathrm{p}=0.004$ \\
\hline
\end{tabular}


Table 2. Correlation: ITIL implementation progress and total number of employees

\begin{tabular}{|l|l|l|c|c|}
\hline Correlation & & $\begin{array}{c}\text { Number of } \\
\text { employees }\end{array}$ & $\begin{array}{c}\text { ITIL } \\
\text { Implementation } \\
\text { Progress }\end{array}$ \\
\hline Spearman's rho & Total Staff & Correlation Coefficient & 1.000 & $.523^{* *}$ \\
\cline { 3 - 5 } & Sig. (1-tailed) &. & .000 \\
\hline **. Correlation is significant at the 0.01 level (1-tailed). \\
Spearman rho=0.523, p=0.000
\end{tabular}

Table 3. Correlation: ITIL implementation progress and total number of IT staff

\begin{tabular}{|l|l|l|l|c|}
\hline Correlation & & $\begin{array}{l}\text { ITIL Implementation } \\
\text { Progress }\end{array}$ & $\begin{array}{l}\text { Total IT } \\
\text { Staff }\end{array}$ \\
\hline Spearman's rho & $\begin{array}{l}\text { ITIL } \\
\text { Implementation } \\
\text { Progress }\end{array}$ & $\begin{array}{l}\text { Correlation } \\
\text { Coefficient }\end{array}$ & 1.000 & $.555^{* *}$ \\
\cline { 2 - 4 } & Sig. (1-tailed) & .000 \\
\hline $\begin{array}{l}* * \text {. Correlation is significant at the } 0.01 \text { level (1-tailed). } \\
\text { Spearman rho }=0.555, \mathrm{p}=0.000\end{array}$
\end{tabular}

